\title{
Stochastic Resonance of Ensemble Neurons for Transient Spike Trains: A Wavelet Analysis
}

\author{
Hideo Hasegawa * \\ Department of Physics, Tokyo Gakugei University, Koganei, Tokyo 184-8501, Japan
}

(October 25, 2018)

\begin{abstract}
By using the wavelet transformation (WT), we have analyzed the response of an ensemble of $N(=1,10,100$ and 500) Hodgkin-Huxley (HH) neurons to transient $M$-pulse spike trains $(M=1-3)$ with independent Gaussian noises. The cross-correlation between the input and output signals is expressed in terms of the WT expansion coefficients. The signal-to-noise ratio (SNR) is evaluated by using the denoising method within the WT, by which the noise contribution is extracted from output signals. Although the response of a single $(N=1)$ neuron to sub-threshold transient signals with noises is quite unreliable, the transmission fidelity assessed by the cross-correlation and SNR is shown to be much improved by increasing the value of $N$ : a population of neurons play an indispensable role in the stochastic resonance (SR) for transient spike inputs. It is also shown that in a large-scale ensemble, the transmission fidelity for supra-threshold transient spikes is not significantly degraded by a weak noise which is responsible to SR for sub-threshold inputs.
\end{abstract}

PACS No. 87.10.+e 84.35.+i 05.45.-a 07.05.Mh

*E-mail: hasegawa@u-gakugei.ac.jp 


\section{INTRODUCTION}

In recent years much studies have been made for the stochastic resonance (SR), in which weak input signals are enhanced by background noises [1]- [2]. This paradoxical SR phenomenon was first discovered in the context of climate dynamics, and it is now reported in many non-linear systems such as electric circuits, ring lasers, semiconductor devices and neurons.

For single neurons, SR has been studied by using various theoretical models such as the integrate-and-fire (IF) model [3]- [5], the FitzHough-Nagumo (FN) model [6]- [8] and the Hodgkin-Huxley $(\mathrm{HH})$ model [9]. In these studies, a weak periodic (sinusoidal) signal is applied to the neuron, and it has been reported that the peak height of the interspikeinterval (ISI) distribution [3]- [6] or the signal-to-noise ratio (SNR) of output signals [7]- 9] shows the maximum when the noise intensity is changed.

$\mathrm{SR}$ in coupled or ensemble neurons has been also investigated by using the IF model [10][11], FN model [12]- 14] and HH model [15]- 18]. The transmission fidelity is examined by calculating various quantities: the peak-to-width ratio of output signals [12] [10] [15] [16], the cross-correlation between input and output signals [10] [18], SNR [10] [13] [18], and the mutual information [14]. One or some of these quantities has been shown to take a maximum as functions of the noise intensity and the coupling strength. Collins, Chow and Imhoff [12] have pointed out that SR of ensemble neurons is improved as the size of an ensemble is increased. Some physiological experiments support SR in real, biological systems of crayfish [19] 20, cricket 21] and rat 22] 23].

SR studies mentioned above are motivated from the fact that peripheral sensory neurons play a role of transducers, receiving analog stimula and emitting spikes. In central neural systems, however, cortical neurons play a role of data-processors, receiving and transmitting spike trains. The possibility of $\mathrm{SR}$ in the spike transmission has been reported [24]- 26]. The response to periodic coherent spike-train inputs has been shown to be enhanced by an addition of weak spike-train noises whose interspike intervals (ISIs) have the Poison or gamma distribution.

It should be stressed that these theoretical studies on SR in neural systems have been performed mostly for stationary analog (or spike-train) signals although they are periodic or aperiodic [27]. There has been few theoretical studies on SR for non-stationary signals. Fakir [28] has discussed SR for non-stationary analog inputs with finite duration by calculating the cross-correlation. By applying a single impulse, Pei, Wilkinson and Moss [15] have demonstrated that the spike-timing precision is improved by noises in an ensemble of 1000 $\mathrm{HH}$ neurons. One of the reason why SR study for stationary signals is dominant, is mainly due to the fact that the stationary signals can be easily analyzed by the Fourier transformation (FT) with which, for example, the SNR is evaluated from FT spectra of output signals. The FT requires that a signal to be examined is stationary, not giving the time evolution of the frequency pattern. Actual biological signals are, however, not necessarily stationary. It has been reported that spike signals in cortical neurons are generally not stationary, rather they are transient signals or bursts [29], whereas periodic spikes are found in systems such as auditory systems of owl [30] and the electrosensory system of electric fish [31].

The limitation of the FT analysis can be partly resolved by using the short-time Fourier transformation (STFT). Assuming that the signal is quasi-stationary in the narrow time 
period, the FT is applied with time-evolving narrow windows. Then STFT yields the time evolution of the frequency spectrum. The STFT, however, has a critical limitation violating the uncertainty principle, which asserts that if the window is too narrow, the frequency resolution will be poor whereas if the window is too wide, the time resolution will be less precise. This limitation becomes serious for signals with much transient components, like spike signals.

The disadvantage of the STFT is overcome in the wavelet transformation (WT) [32]. In contrast to the FT, the WT offers the two-dimensional expansion for a time-dependent signal with the scale and translation parameters which are interpreted physically as the inverse of frequency and time, respectively. As a basis of the WT, we employ the mother wavelet which is localized in both frequency and time domains. The WT expansion is carried out in terms of a family of wavelets which is made by dilation and translation of the mother wavelet. The time evolution of frequency pattern can be followed with an optimal time-frequency resolution.

The WT appears to be an ideal tool for analyzing signals of a non-stationary nature. In recent years the WT has been applied to an analysis of biological signals [33], such as electoencephalographic (EEG) waves [34- [40], and spikes [41]- [45]. EEG is a reflection of the activity of ensembles of neurons producing oscillations. By using the WT, we can obtain the time-dependent decomposition of EEG signals to $\delta(0.3-3.5 \mathrm{~Hz}), \theta(3.5-7.5 \mathrm{~Hz}), \alpha(7.5-$ $12.5 \mathrm{~Hz}), \beta(12.5-30.0 \mathrm{~Hz})$ and $\gamma(30-70 \mathrm{~Hz})$ components [34- [40]. It has been shown that the WT is a powerful tool to the spike sorting in which coherent signals of a single target neuron are extracted from mixture of response signals [41]- 433. Quite recently Hasegawa [45] has made an analysis of transient spike-train signals of a $\mathrm{HH}$ neuron with the use of WT, calculating the energy distribution and Shanon entropy.

It is interesting to analyze the response of ensemble neurons to transient spike inputs in noisy environment by using the WT. There are several sources of noises: (i) cells in sensory neurons are exposed to noises arising from the outer world, (ii) ion channels of the membrane of neurons are known to be stochastic [46], (iii) the synaptic transmission yields noises originating from random fluctuations of the synaptic vesicle release rate [47, and (iv) synaptic inputs include leaked currents from neighboring neurons [48]. Most of existing studies on SR adopt the Gaussian noises, taking account of the items (i)-(iii). Simulating the noise of the item (iv), Refs. [24]- [26] include spike-train noises whose ISIs have the Poisson or gamma distribution. In this study we take into account Gaussian noises; SR for spike-train inputs with added spike-train noises will be discussed in a separate paper [49].

We assume an ensemble of Hodgkin-Huxley (HH) neurons to receive transient spike trains with independent Gaussian noises. The HH neurons model is adopted because it is known to be the most realistic among theoretical models [50]. The signal transmission is assessed by the cross-correlation between input and output signals and SNR, which are expressed in terms of WT expansion coefficients. In calculating the SNR, we adopt the denoising technique within the WT method [51]- [54], by which the noise contribution is extracted from output signals.

Our paper is organized as follows: In Sec. IIA, an adopted model for an ensemble of $\mathrm{N}$-unit HH neurons is described, and in Sec. IIB the WT is briefly discussed. We present the calculated results for $M=1$ pulse train in Sec. IIIA, and the results for spike trains with $M=2$ and 3 are discussed in Sec.IIB. The final Sec. IV is devoted to conclusion and 
discussion.

\section{CALCULATION METHODS}

\section{A. Ensemble Neuron Model}

We assume a network consisting of $N$-unit $\mathrm{HH}$ neurons which receive the same spike trains but independent Gaussian white noises through excitatory synapses. Spikes emitted by the ensemble neurons are collected by a summing neuron. A similar model was previously adopted by several authors studying SR for analog signals [12] [15] [16]. An input signal in this paper is a transient spike train consisting of $M$ impulses $(M=1-3)$. Dynamics of the membrane potential $V_{i}$ of the $\mathrm{HH}$ neuron $i$ is described by the non-linear differential equations given by

$$
\bar{C} d V_{i}(t) / d t=-I_{i}^{\mathrm{ion}}+I_{i}^{\mathrm{ps}}+I_{i}^{\mathrm{n}}, \quad(\text { for } 1 \leq i \leq N)
$$

where $\bar{C}=1 \mu \mathrm{F} / \mathrm{cm}^{2}$ is the capacity of the membrane. The first term $I_{i}^{\text {ion }}$ of Eq.(1) denotes the ion current given by

$$
I_{i}^{\text {ion }}=g_{\mathrm{Na}} m_{i}^{3} h_{i}\left(V_{i}-V_{\mathrm{Na}}\right)+g_{\mathrm{K}} n_{i}^{4}\left(V_{i}-V_{\mathrm{K}}\right)+g_{\mathrm{L}}\left(V_{i}-V_{\mathrm{L}}\right),
$$

where the maximum values of conductivities of $\mathrm{Na}$ and $\mathrm{K}$ channels and leakage are $g_{\mathrm{Na}}=$ $120 \mathrm{mS} / \mathrm{cm}^{2}, g_{\mathrm{K}}=36 \mathrm{mS} / \mathrm{cm}^{2}$ and $g_{\mathrm{L}}=0.3 \mathrm{mS} / \mathrm{cm}^{2}$, respectively; the respective reversal potentials are $V_{\mathrm{Na}}=50 \mathrm{mV}, V_{\mathrm{K}}=-77 \mathrm{mV}$ and $V_{\mathrm{L}}=-54.5 \mathrm{mV}$. Dynamics of the gating variables of $\mathrm{Na}$ and $\mathrm{K}$ channels, $m_{i}, h_{i}$ and $n_{i}$, are described by the ordinary differential equations, whose details have been given elsewhere [50] [56].

The second term $I_{i}^{\mathrm{ps}}$ in Eq.(1) denotes the postsynaptic current given by

$$
I_{i}^{\mathrm{ps}}=\sum_{m=1}^{M} g_{s}\left(V_{a}-V_{s}\right) \alpha\left(t-t_{\mathrm{i} m}\right),
$$

which is induced by an input spike with the magnitude $V_{a}$ given by

$$
U_{i}(t)=V_{a} \sum_{m=1}^{M} \delta\left(t-t_{\mathrm{i} m}\right),
$$

with the alpha function $\alpha(t)$ :

$$
\alpha(t)=\left(t / \tau_{\mathrm{s}}\right) e^{-t / \tau_{\mathrm{s}}} \Theta(t)
$$

In Eqs.(3)-(5) $t_{\mathrm{i} m}$ is the $m$-th firing time of the input, the Heaviside function is defined by $\Theta(t)=1$ for $x \geq 0$ and 0 for $x<0$, and $g_{s}, V_{\mathrm{s}}$ and $\tau_{\mathrm{s}}$ stand for the conductance, reversal potential and time constant, respectively, of the synapse.

The third term $I_{i}^{\mathrm{n}}$ in Eq.(1) denotes the Gaussian noise given by

$$
<\overline{I_{i}^{n}(t)}>=0
$$




$$
<\overline{I_{i}^{n}(t) I_{\ell}^{n}\left(t^{\prime}\right)}>=2 D \delta_{i \ell} \delta\left(t-t^{\prime}\right),
$$

where the overline $\bar{X}$ and the bracket $\langle X\rangle$ denote the temporal and spatial averages, respectively, and $D$ the intensity of white noises.

The output spike of the neuron $i$ in an ensemble is given by

$$
U_{\mathrm{o} i}(t)=V_{a} \sum_{n} \delta\left(t-t_{\mathrm{oin}}\right),
$$

in a similar form as an input spike [Eq.(4)], where $t_{\text {oin }}$ is the $n$-th firing time when $V_{i}(t)$ crosses $V_{z}=0 \mathrm{mV}$ from below.

We should remark that our model given by Eqs.(1)-(7) does not include couplings among ensemble neurons. This is in contrast with some works on ensemble neurons [13] [14] [17] [18] where introduced couplings among neurons play an important role in SR besides noises, related discussion being given in Sec. IV.

Differential equations given by Eqs.(1)-(7) are solved by the forth-order Runge-Kutta method by the integration time step of $0.01 \mathrm{~ms}$ with double precision. Some results are examined by using the exponential method. The initial conditions for the variables are given by $V_{i}(t)=-65 \mathrm{mV}, m_{i}(t)=0.0526, h_{i}(t)=0.600, n_{i}(t)=0.313$ at $t=0$, which are the rest-state solution of a single $\mathrm{HH}$ neuron. Hereafter time, voltage, conductance, current, and $D$ are expressed in units of $\mathrm{ms}, \mathrm{mV}, \mathrm{mS} / \mathrm{cm}^{2}, \mu \mathrm{A} / \mathrm{cm}^{2}$, and $\mu \mathrm{A}^{2} / \mathrm{cm}^{4}$, respectively. We have adopted parameters of $V_{a}=30, V_{c}=-50$, and $\tau_{\mathrm{s}}=\tau_{n}=2$. Adopted values of $g_{s}, D$, $M$ and $N$ will be described shortly.

\section{B. Wavelet Analysis}

There are two types of WTs: one is the continuous wavelet transformation (CWT) and the other the discrete wavelet transformation (DWT). In the former the parameters denoting the scale and trasnlation are continuous variables while in the latter they are discrete variables.

The CWT for a given regular function $f(t)$ is defined by

$$
c(a, b)=\int \mathrm{d} t \psi_{a b}^{*}(t) f(t) \equiv<\psi_{a b}(t), f(t)>,
$$

with a family of wavelets $\psi_{a b}(t)$ generated by

$$
\psi_{a b}(t)=|a|^{-1 / 2} \psi\left(\frac{t-b}{a}\right),
$$

where $\psi(t)$ is the mother wavelet, the star denotes the complex conjugate, and $a$ and $b$ express the scale change and translation, respectively, and they physically stand for the inverse of the frequency and the time. Then the CWT transforms the time-dependent function $f(t)$ into the frequency- and time-dependent function $c(a, b)$. The mother wavelet is a smooth function with good localization in both frequency and time spaces. A wavelet family given by Eq.(10) plays a role of elementary function, representing the function $f(t)$ as a superposition of wavelets $\psi_{a b}(t)$.

The inverse of the wavelet transformation may be given by 


$$
f(t)=C_{\psi}^{-1} \int \frac{\mathrm{d} a}{a^{2}} \int \mathrm{d} b c(a, b) \psi_{a b}(t),
$$

when the mother wavelet satisfies the following two conditions:

(i) the admissibility condition given by

$$
0<C_{\psi}<\infty
$$

with

$$
C_{\psi}=\int_{-\infty}^{\infty} \mathrm{d} \omega|\hat{\Psi}(\omega)|^{2} /|\omega|
$$

where $\hat{\Psi}(\omega)$ is the Fourier transformation of $\psi(t)$, and

(ii) the zero mean of the mother wavelet:

$$
\int_{-\infty}^{\infty} \mathrm{d} t \psi(t)=\hat{\Psi}(0)=0
$$

On the contrary, the DWT is defined for discrete values of $a=2^{j}$ and $b=2^{j} k(j, k$ : integers) as

$$
c_{j k} \equiv c\left(2^{j}, 2^{j} k\right)=<\psi_{j k}(t), f(t)>
$$

with

$$
\psi_{j k}(t)=2^{-j / 2} \psi\left(2^{-j} t-k\right) .
$$

The ortho-normal condition for the wavelet functions is given by

$$
<\psi_{j k}(t), \psi_{j^{\prime} k^{\prime}}(t)>=\delta_{j j^{\prime}} \delta_{k k^{\prime}},
$$

which leads to the inverse DWT:

$$
f(t)=\sum_{j} \sum_{k} c_{j k} \psi_{j k}(t)
$$

In the multiresolution analysis (MRA) of the DWT, we introduce a scaling function $\phi(t)$, which satisfies the recurrent relation with $2 K$ masking coefficients, $h_{k}$, given by

$$
\phi(t)=\sqrt{ } 2 \sum_{k=0}^{2 K-1} h_{k} \phi(2 t-k),
$$

with the normalization condition for $\phi(t)$ given by

$$
\int \mathrm{dt} \phi(t)=1
$$

A family of wavelet functions is generated by

$$
\psi(t)=\sqrt{ } 2 \sum_{k=0}^{2 K-1}(-1)^{k} h_{2 K-1-k} \phi(2 t-k) .
$$


The scaling and wavelet functions satisfy the ortho-normal relations:

$$
\begin{aligned}
& <\phi(t), \phi(t-m)>=\delta_{m 0} \\
& <\psi(t), \psi(t-m)>=\delta_{m 0} \\
& <\phi(t), \psi(t-m)>=0 .
\end{aligned}
$$

A set of masking coefficients $h_{j}$ is chosen so as to satisfy the conditions shown above.

The simplest wavelet function for $K=1$ is the Harr wavelet for which we get $h_{0}=h_{1}=$ $1 / \sqrt{ } 2$, and

$$
\begin{aligned}
\psi_{\mathrm{H}}(t) & =1, \quad \text { for } 0 \leq t<1 / 2 \\
& =-1, \quad \text { for } 1 / 2 \leq t<1 \\
& =0, \quad \text { otherwise }
\end{aligned}
$$

In the more sophisticated wavelets like the Daubechies wavelet, an additional condition given by

$$
\int \mathrm{d} t t^{\ell} \psi(t)=0, \quad \text { for } \ell=0,1,2,3 \ldots . L-1
$$

is imposed for the smoothness of the wavelet function. Furthermore, in the Coiflet wavelet, for example, a similar smoothing condition is imposed also for the scaling function as

$$
\int \mathrm{d} t t^{\ell} \phi(t)=0, \quad \text { for } \ell=1,2,3 \ldots . L^{\prime}-1
$$

Once WT coefficients are obtained, we can calculate various quantities such as autoand cross-correlations and SNR, as will be discussed shortly. In principle the expansion coefficients $c_{j k}$ in DWT may be calculated by using Eqs.(15) and (16) for a given function $f(t)$ and an adopted mother wavelet $\psi(t)$. This integration is, however, inconvenient, and in an actual fast wavelet transformation, the expansion coefficients are obtained by a matrix multiplication with the use of the iterative formulae given by the masking coefficients and expansion coefficients of the neighboring levels of indices, $j$ and $k$ [32].

One of the advantages of the WT over FT is that we can choose a proper mother wavelet among many mother wavelets, depending on a signal to be analyzed. Among many candidates of mother wavelets, we have adopted the Coiflet with level 3, compromising the accuracy and the computational effort. The WT has been performed by using the MATLAB wavelet tool box.

\section{CALCULATED RESULTS}

\section{A. Input Pulses with $M=1$}

Firstly we discuss the case in which ensemble $\mathrm{HH}$ neurons receive a common single $(M=1)$ impulse. When input synaptic strength is small: $g_{s}<g_{t h}$, no neurons fire in the noise-free case, while it is sufficiently large $\left(g_{s} \geq g_{t h}\right)$ neurons fire, where $g_{t h}=0.091$ is the threshold value. For a while, we will discuss the sub-threshold case of $g_{s}=0.06<g_{t h}$ with 
$N=500$. The $M=1$ input pulse is applied at $t=100 \mathrm{~ms}$, as shown in Fig. 1(a). Figure (b) shows the time dependence of the postsynaptic current $I_{i}=I_{i}^{\mathrm{ps}}+I_{i}^{\mathrm{n}}$ of the neuron $i=1$ with added noises of $D=0.5$. Because neurons receive noises for $0 \leq t<100 \mathrm{~ms}$, the states of neurons when they receive input pulse are randomized [16]. Figure 1(c) shows the time dependence of the membrane potential $V_{1}$ of the neuron 1 , which fires with a delay of about $6 \mathrm{~ms}$. This delay is much larger than the conventional value of 2-3 $\mathrm{ms}$ for the supra-threshold inputs because the integration of marginal inputs at synapses needs a fairly a long period before firing [56]. Firings in ensemble 500 neurons for $D=0.5$ are depicted by raster in Fig. 2(a). We note that neurons fire not only by input pulses plus noises but also spuriously by noises only. When the noise intensity is increased to $D=1.0$, spurious firings are much increased, as Fig. 2(b) shows.

We assume that information is carried by firing times of spikes but not by details of their shapes. In order to study how information is transmitted through ensemble $\mathrm{HH}$ neurons with the use of the DWT, we divide the time scale by the width of time bin of $T_{\mathrm{b}}$ as $t=t_{\ell}=\ell T_{\mathrm{b}}$ $(\ell$ : integer $)$, and define the input and output signals within the each time bin by

$$
\begin{gathered}
W_{\mathrm{i}}(t)=\sum_{m=1}^{M} \Theta\left(\left|t-t_{\mathrm{i} m}\right|-T_{\mathrm{b}} / 2\right), \\
W_{\mathrm{o}}(t)=(1 / N) \sum_{i=1}^{N} \sum_{n} \Theta\left(\left|t-t_{\mathrm{o} i n}\right|-T_{\mathrm{b}} / 2\right) .
\end{gathered}
$$

In Eqs. (28) and (29) $\Theta(t)$ stands for the Heaviside function, $W_{\mathrm{i}}(t)$ the external input signal (without noises), $W_{\mathrm{o}}(t)$ the output signal averaged over the ensemble neurons, $t_{\mathrm{i} m}$ the $m$-th firing time of inputs, and $t_{\text {oin }}$ the $n$-th firing time of outputs of the neuron $i$ [Eq.(8)]. The time bin is chosen as $T_{\mathrm{b}}=5 \mathrm{~ms}$ in our simulations. A single simulation has been performed for $320\left(=2^{6} T_{\mathrm{b}}\right)$ ms. Figures $1(\mathrm{~d})$ and $1(\mathrm{e})$ show the time dependence of $W_{\mathrm{i}}(t)$ and $W_{\mathrm{o}}(t)$, respectively, for the case of $D=0.5$. The magnitude of $W_{\mathrm{o}}(t)$ is much smaller than that of $W_{\mathrm{i}}(t)$ because only a few neurons fire among 500 neurons. The peak position of $W_{\mathrm{o}}(t)$ is slightly shifted compared with that of $W_{\mathrm{i}}(t)$ because of a significant delay of neuron firings as mentioned above.

\section{Wavelet Transformation}

Now we apply the DWT to input and output signals . By substituting $f(t)=W_{\mathrm{i}}(t)$ or $W_{\mathrm{o}}(t)$ in Eq.(15), we get their WT coefficients given by

$$
c_{\lambda j k}=\int \mathrm{d} t \psi_{j k}^{*}(t) W_{\lambda}(t), \quad(\lambda=\mathrm{i}, \mathrm{o})
$$

where $\psi_{j k}(t)$ is a family of wavelets generated from the mother Coiflet wavelet [see Eq.(16)]. The uppermost frame of Fig. 3(a) expresses the input signal $W_{\mathrm{i}}(t)$. Note that the lower and upper horizontal scales express $b$ and $b T_{b}$ (in units of ms), respectively. Figure 3(b) shows the calculated WT coefficients of $W_{\mathrm{i}}(t)$ which are plotted as a function of $b(j)=(k-0.5) 2^{j}$ for various $j$ values after convention. The WT decomposition of the signal: $f(t)=\sum_{j=1}^{5} f_{j}(t)$, is plotted in Fig. 3(a). The WT coefficients of $j=1$ and 2 have large values near $b=$ 
$20\left(b T_{b}=100 \mathrm{~ms}\right)$ where $W_{\mathrm{i}}(t)$ has a peak. Contributions from $j=1$ and $j=2$ are predominant in $W_{\mathrm{i}}(t)$. It is noted that the WT coefficient for $j=4$ has a significant value at $b \sim 56$ far away from $b=20$. The WT decomposition and WT coefficients for output signal $W_{\mathrm{o}}(t)$ for $D=0.5$ are shown in Fig. 4(a) and 4(b), respectively. The dominant contribution arises from $j=1$ in $W_{\mathrm{o}}(t)$. Similar plots of the WT decomposition and the WT coefficients of the output signal $W_{\mathrm{o}}(t)$ for $D=1.0$ are presented in Figs. 5(a) and 5(b), respectively. As the noise intensity is increased, fine structures in the WT coefficients appear, in particular for small $j$.

Auto- and Cross-correlations

The auto-correlation functions for input and output signals are defined by

$$
\begin{aligned}
\Gamma_{\lambda \lambda} & =M^{-1} \int \mathrm{d} t W_{\lambda}(t)^{*} W_{\lambda}(t), \\
& =M^{-1} \sum_{j} \sum_{k} c_{\lambda j k}^{*} c_{\lambda j k}, \quad(\lambda=\mathrm{i}, \mathrm{o})
\end{aligned}
$$

where the ortho-normal relations of the wavelets given by Eqs.(22)-(24) are employed. Similarly the cross-correlation between input and output signals is defined by

$$
\begin{aligned}
\Gamma_{i o}(\beta) & =M^{-1} \int \mathrm{d} t W_{i}(t)^{*} W_{o}\left(t+\beta T_{\mathrm{b}}\right), \\
& =M^{-1} \sum_{j} \sum_{k} c_{\mathrm{i} j k}^{*} c_{\mathrm{o} j k}(\beta),
\end{aligned}
$$

where $c_{\mathrm{i} j k}$ and $c_{\mathrm{o} j k}(\beta)$ are the expansion coefficients of $W_{i}(t)$ and $W_{o}\left(t+\beta T_{\mathrm{b}}\right)$, respectively. The maxima in the cross-correlation and the normalized one are given by

$$
\begin{gathered}
\Gamma=\max _{\beta}\left[\Gamma_{\mathrm{io}}(\beta)\right], \\
\gamma=\max _{\beta}\left[\frac{\Gamma_{\mathrm{io}}(\beta)}{\sqrt{ } \Gamma_{\mathrm{ii}} \sqrt{ } \Gamma_{\mathrm{oo}}}\right] .
\end{gathered}
$$

It is noted that for the suprashreshold inputs in the noise-free case, we get $\Gamma_{\mathrm{ii}}=\Gamma_{\mathrm{oo}}=\Gamma_{\mathrm{io}}=1$ and then $\Gamma=\gamma=1$.

Figure 6(a) shows the $D$ dependence of $\Gamma, \Gamma_{\text {oo }}$ and $\gamma$ for $g=0.06$ and $N=500$. They are zero at $D=0$ because of the adopted sub-threshold parameters. As increasing $D$ from zero, $\Gamma$ and $\Gamma_{\text {oo }}$ are gradually increased. Because of the factor of $1 / \sqrt{ } \Gamma_{\text {oo }}$ in Eq.(36), the magnitude of $\gamma$ is larger than those of $\Gamma$ and $\Gamma_{\text {oo }}$. We note that $\gamma$ is enhanced by weak noises and it is decreased at larger noises, which is a typical SR behavior.

\section{Signal-to-Noise Ratio}

We will evaluate the SNR by employing the denoising method [51] [54. The key point in the denoising is how to choose which wavelet coefficients are correlated with the signal and

which ones with noises. The simple denoising method is to neglect some DWT expansion 
coefficients when reproducing the signal by the inverse wavelet transformation. We get the denoising signal by the inverse WT [Eq.(18)]:

$$
W_{\lambda}^{d n}(t)=\sum_{j} \sum_{k} c_{\lambda j k}^{d n} \psi_{j k}(t)
$$

with the desnoising WT coefficients $c_{j k}^{d n}$ to be chosen properly as will be discussed below. The simplest denoising method, for example, is to assume that WT components for $a<a_{c}$ in the $(a, b)$ plane arise from noises to set the denoising WT coefficients as

$$
\begin{array}{rlrl}
c_{j k}^{d n} & =c_{j k}, \quad & \text { for } j \geq j_{c}\left(a \geq a_{c}\right) \\
& =0, \quad \text { otherwise }
\end{array}
$$

where $j_{c}\left(=\log _{2} a_{c}\right)$ is the critical $j$ value [51.

In this study we adopt a more sophisticated method, assuming that the components for $b<b_{\mathrm{L}}$ or $b>b_{\mathrm{U}}$ at $a<a_{c}$ in the $(\mathrm{a}, \mathrm{b})$ plane are noises to set the denoising WT coefficients as

$$
\begin{aligned}
c_{j k}^{d n} & =c_{j k}, & & \text { for } j \geq j_{c} \text { or } k_{\mathrm{L}} \leq k \leq k_{\mathrm{U}} \\
& =0, & & \text { otherwise }
\end{aligned}
$$

where $j_{c}\left(=\log _{2} a_{c}\right)$ denotes the critical $j$ value, and $k_{\mathrm{L}}\left(=b_{\mathrm{L}} 2^{-j}\right)$ and $k_{\mathrm{U}}\left(=b_{\mathrm{U}} 2^{-j}\right)$ are the lower and upper critical $k$ values, respectively. We get the inverse, denoising signal by using Eq.(37) with the denoising WT coefficients determined by Eq.(39).

From the above consideration, we may define the signal and noise components by

$$
\begin{gathered}
A_{s}=\int \mathrm{d} t W_{\mathrm{o}}^{d n}(t)^{*} W_{\mathrm{o}}^{d n}(t), \\
=\sum_{j} \sum_{k}\left|c_{\mathrm{o} j k}^{d n}\right|^{2}, \\
A_{n}=\int \mathrm{d} t\left[W_{\mathrm{o}}(t)^{*} W_{\mathrm{o}}(t)-W_{\mathrm{o}}^{d n}(t)^{*} W_{\mathrm{o}}^{d n}(t)\right], \\
=\sum_{j} \sum_{k}\left(\left|c_{\mathrm{o} j k}\right|^{2}-\left|c_{\mathrm{o} j k}^{d n}\right|^{2}\right),
\end{gathered}
$$

The SNR is defined by

$$
S N R=10 \log _{10}\left(A_{s} / A_{n}\right) \quad(\mathrm{dB}) .
$$

In the present case we can fortunately obtain the WT coefficients for ideal case of noisefree and supra-threshold inputs. We then properly determine the denoising parameters of $j_{c}, b_{L}$ and $b_{U}$. From the observation of the WT coefficients for the ideal case, which is not shown here but is not dissimilar to those shown in Figs. 3(a) and 4(a), we assume that the upper and lower bounds, may be chosen as

$$
b_{\mathrm{L}}=t_{\mathrm{o} 1} / T_{\mathrm{b}}-\delta b, \quad b_{\mathrm{U}}=t_{\mathrm{o} M} / T_{\mathrm{b}}+\delta b,
$$


where $t_{\mathrm{o} 1}\left(t_{\mathrm{o} M}\right)$ are the first $(M$-th) firing times of output signals in the ideal case of noisefree and supra-threshold inputs, and $\delta b$ denotes the marginal distance from the $b$ values expected to be responsible to the signal transmission.

The denoising WT coefficients $c_{j k}^{\mathrm{dn}}$ of $W_{\mathrm{o}}$ for $D=0.5$ and $D=1.0$ are denoted by dashed bars in Fig. 4(b) and 5(b), respectively. Note that the positions of dashed bars are slightly shifted leftward to avoid the superposition with the solid bars representing the original WT coefficients. In the case of $D=0.5$, the denoising WT coefficients are almost the same as the original ones. The inverse WT signal of $W_{\mathrm{o}}^{\mathrm{dn}}$, which is given by Eq.(37) and which is shown by the dashed curve in Fig. 4(a), is almost the same as the original signal shown by the solid curve. On the contrary, in the case of $D=1.0$, the denoising WT coefficients of $W_{\mathrm{o}}$ do not include the terms far away from $b=20$ for $j<j_{c}$ in the original WT coefficients. The inverse WT signal of $W_{\mathrm{o}}^{d n}$, shown by the dashed curve in Fig. 5(a), has less amount of ripples than the original signal shown by the solid curve.

Figure $7(\mathrm{a})$ and $7(\mathrm{~b})$ show the calculated SNR of $W_{\mathrm{o}}$ as a function of $\delta b$ for various $j_{c}$ values with $D=0.5$ and $D=1.0$, respectively. We note that the value of $\mathrm{SNR}$ is rather insensitive to a choice of the parameters of $\delta b$ and $j_{c}$. Then we have decided to adopt $\delta b=5$ and $j_{c}=3$ for our simulations.

Figure $6(\mathrm{~b})$ shows the $D$ dependence of SNR calculated for $g=0.06$ and $N=500$. We note that SNR shows a typical SR behavior: a rapid rise to a clear peak and a slow decrease for larger value of $D$.

So far our simulation has been made for the fixed parameters of $g_{s}=0.06$ and $N=500$, which will be changed in the followings. Figure $8(\mathrm{a})$ and $8(\mathrm{~b})$ show the $D$ dependence of the cross-correlation $\gamma$ and SNR, respectively, calculated for $N=1,10100$ and 500. The results for $N=1$ and 10 are averaged values of ten and hundred trials, respectively, their root-mean-square (RMS) values being shown by error bars in Fig. 8(a) and 8(b). The results of $N=500$ show the typical SR behavior as mentioned before [Figs. 6(a) and 6(b)]. On the contrary, SR effect for a single $(N=1)$ is marginally realized in SNR but not in $\gamma$. Large error bars for the results of $N=1$ implies that the reliability of information transmission is very low in the sub-threshold condition [57]. This is clearly seen in Fig. 9(a) and 9(b) where $\gamma$ and SNR for $N=1$ are plotted against the trial number of 100 runs. The signal can be transmitted only when the signal fortunately coincides with noises and the signal plus noise crosses the threshold level. Then only 13 among 100 trials are succeeded in the transmission of $M=1$ inputs through a neuron. We note in Figs. 8(a) and 8(b) that as the size of the network is much increased, the peak values of $\gamma$ and SNR are much enhanced and the SR behavior becomes more evident. Figures 8(a) and 8(b) demonstrate that the ensemble of neurons play an indispensable role for information transmission of transient spike signals. This is consistent with the results of Collins et al. [12] and Pei et al. [15], who have pointed out the improvement of the information transmission by increasing the size of the network.

Next we change the value of $g_{s}$, the strength of input synapses. The $g_{s}$ dependence of $\gamma$ and SNR for $N=500$ neurons is shown in Figs. 10(a) and 10(b), respectively, where values of $D=0.0,0.5$ and 1.0 are employed. Note that in the noise-free case $(D=0)$, we get $\gamma=1$ and $\mathrm{SNR}=\infty$ for $g_{s} \geq g_{\mathrm{th}}$ but $\gamma=0$ and $\mathrm{SNR}=-\infty$ for $g_{s}<g_{\mathrm{th}}$, as shown by dotted curves in Figs. 10(a) and 10(b). It is shown that moderate sub-threshold noises considerably improve the transition fidelity. We also note that the presence of such noises 
does not significantly degrade the transmission fidelity for supra-threshold cases in ensemble neurons. For a comparison, the $g_{s}$ dependence of $\gamma$ and SNR for single $(N=1)$ neurons is shown in Figs. 11(a) and 11(b), respectively. As having been shown in Fig. 8(a), SR for $N=1$ is less significant compared to that for $N=500$.

\section{B. Input Pulses with $M=2$ and 3}

Now we discuss the cases of $M=2$ and 3 . Input pulses are applied at $t=100$ and 125 ms for the $M=2$ case. The input ISI is assume to be $T_{\mathrm{i}}=25 \mathrm{~ms}$ because spikes with this value of ISI are reported to be ubiquitous in cortical brains [29]. Firings of 500 neurons for the noise intensity of $D=1.0$ are shown by raster in Figs. 12(a), which shows that firings occur mainly at $t \sim 103$ and $128 \mathrm{~ms}$ with a delay of about $3 \mathrm{~ms}$. The output signal $W_{\mathrm{o}}(t)$ averaged over $N=500$ neurons is depicted by the solid curve in the uppermost frame of Fig. 13(a), which has main two peaks. The WT decomposition and WT coefficients of $W_{\mathrm{o}}(t)$ are plotted in Figs. 13(a) and 13(b), respectively. When we compare the results for $M=2$ shown in Figs. 13(a) and 13(b) with those for $M=1$ shown in Fig. 5(a) and 5 (b), we note that components for $j=1$ and 2 for $M=2$ are much increased because of the presence of the second peak for $M=2$ inputs while the contributions from $j \geq 3$ are changed little. The denoising has been made by the procedure given by Eqs. (37), (39) and (45). The denoising WT coefficients are plotted by dashed bars in Fig. 13(b) and the denoising component signals are shown by dashed curves in Fig. 13(a).

We apply the $M=3$ pulse at $t=100,125$ and $150 \mathrm{~ms}$, the input ISI being again $T_{\mathrm{i}}=25$ ms. Raster in Fig. 12(b) shows firing of 500 neurons for $D=1.0$. Firings mainly occur at $t \sim 103,128$ and $153 \mathrm{~ms}$ with a delay of $3 \mathrm{~ms}$. The solid curve in the uppermost frame of Fig. 14(a) shows the averaged output of $W_{\mathrm{o}}(t)$ for $D=1.0$. The sold bars in Fig. 14(b) show its WT coefficients and the solid curves in Fig. 14(a) express its WT decomposition. Again components of $j=1$ and 2 are increased because of the second and third peaks in $W_{\mathrm{o}}(t)$. The denoising WT coefficients are shown by dashed bars in Fig. 14(b) and denoising signals by dashed curves in Fig. 14(a).

The calculated $D$ dependence of the cross-correlation $\gamma$ [SNR] for $M=1,2$ and 3 is plotted in Fig. 15(a) [15(b)]. Both $\gamma$ and SNR show typical SR behavior irrespective of the value of $M$, although a slight difference exists between the $M$ dependence of $\gamma$ and SNR: for larger $M$, the former is larger but the latter is smaller at the moderate noise intensity of $D<1.0$. When similar simulations are performed for different ISI values of $T_{\mathrm{i}}=15$ and $35 \mathrm{~ms}$, we obtain results which are almost the same as that for $T_{\mathrm{i}}=25$ (not shown). This is because the output spikes for inputs with $M=2$ and 3 are superposition of an output spike for a $M=1$ input when the ISI is larger than the refractory period of neurons.

\section{CONCLUSION AND DISCUSSION}

It has been controversial how neurons communicate information by action potentials or spikes [58]- 63]. The one issue is whether information is encoded in the average firing rate

of neurons (rate code) or in the precise firing times (temporal code). Since Andrian [64] first noted the relationship between neural firing rate and stimulus intensity, the rate-code 
model has been supported in many experiments of motor and sensory neurons. In the last several years, however, experimental evidences have been accumulated, indicating a use of the temporal code in neural systems [65]- [69]. Human visual systems, for example, have shown to classify patterns within $250 \mathrm{~ms}$ despite the fact that at least ten synaptic stages are involved from retina to the temporal brain [69]. The transmission times between two successive stages of synaptic transmission are suggested to be no more than $10 \mathrm{~ms}$ on the average. This period is too short to allow rates to be determined accurately.

Although much of debates on the nature of the neural code has focused on the rate versus temporal codes, there is the other important issue to consider: information is encoded in the activity of single (or very few) neurons or that of a large number of neurons (population or ensemble code). The population rate code model assumes that information is coded in the relative firing rates of ensemble neurons, and has been adopted in the most of the theoretical analysis. On the contrary, in the population temporal code model, it is assumed that relative timings between spikes in ensemble neurons may be used as an encoding mechanism for perceptional processing [70]- [72]. A number of experimental data supporting this code have been reported in recent years [73]- [76]. For example, data have demonstrated that temporally coordinated spikes can systematically signal sensory object feature, even in the absence of changes in firing rate of the spikes [74].

Our simulations based on the temporal-code model has shown that a population of neurons plays a very important role for the transmission of sub-threshold transient spike signals [Figs. 8(a) and 18(b)]. In particular for single neurons the transmission is quite unreliable and the appreciable SR effect is not realized. When the size of ensemble neurons is increased, the transmission fidelity is much improved in a fairly wide-range of parameters $g_{s}$ including both the sub- and supra-threshold cases (Fig. 10). We note in Figs. 8(a) and 8(b) that $\gamma$ (or SNR) for $N=100$ is different from and larger than that for $N=1$ with 100 trials. This seems strange because a simulation for an ensemble of $N$ neurons is expected to be equivalent to simulations for a single neuron with $N$ trials, if there is no couplings among neurons as in our model. This is, however, not true, and it will be understood as follows. We consider a quantity of $X\left(N, N_{r}\right)$ which is $\gamma$ (or SNR) averaged over $N_{r}$ trials for an ensemble of $N$ neurons. We implicitly express $X\left(N, N_{r}\right)$ as

$$
\begin{aligned}
X\left(N, N_{r}\right) & =\ll F\left(<w_{i}^{(\mu)}>\right) \gg \\
& =\frac{1}{N_{r}} \sum_{\mu=1}^{N_{r}} F\left(\frac{1}{N} \sum_{i=1}^{N} w_{i}^{(\mu)}\right),
\end{aligned}
$$

with

$$
w_{\mathrm{i}}^{(\mu)}=w_{\mathrm{i}}^{(\mu)}(t)=\sum_{n} \Theta\left(\left|t-t_{\mathrm{o} i n}^{(\mu)}\right|-T_{\mathrm{b}} / 2\right),
$$

where $\ll \cdot \gg$ and $\langle\cdot>$ stand for averages over trials and an ensemble neurons, respectively, defined by Eq.(47), $t_{\text {oin }}^{(\mu)}$ is the $n$-th firing time of the neuron $i$ in the $\mu$-th trial, $w_{\mathrm{i}}^{(\mu)}(t)$ is its output signal of the neuron $i$ within a time bin of $T_{\mathrm{b}}[c f$. Eq.(29)], and $F(y(t))$ is a functional of a given function of $y(t)$ relevant to a calculation of $\gamma$ (or SNR). Figures 8(a) and $8(\mathrm{~b})$ show that the relation: $X(100,1)>X(1,100)$, namely

$$
F\left(<w_{i}^{(1)}>\right)>\ll F\left(w_{1}^{(\mu)}\right) \gg,
$$


holds for our $\gamma$ and SNR. Note that if $F(\cdot)$ is linear, we get $X(100,1)=X(1,100)$. This implies that the inequality given by Eq. (49) is expected to arise from a non-linear character of $F(\cdot)$. This reminds us of the algebraic inequality: $f(\langle x\rangle) \geq\langle f(x)\rangle$ valid for a convex function $f(x)$, where the bracket $<\cdot>$ stands for an average over a distribution of a variable $x$. It should be again noted that there is no couplings among our neurons in the adopted model. Then the enhancement of SNR with increasing $N$ is only due to a population of neurons. This is quite different from the result of some papers [13] [14] [17] 18 in which the transmission fidelity is enhanced not only by noises but also by introduced couplings among neurons in an ensemble.

In our simulations reported in Sec. III, independent noises are applied to ensemble neurons. If instead we try to apply the same or completely correlated noise to them, it is equivalent to applying noises to a single neuron, and then appreciable SR effect is not realized as discussed above. Then SR for transient spikes requires independent noises to be applied to a large-scale ensemble of neurons. This is consistent with the result of Liu, $\mathrm{Hu}$ and Wang [18] who discussed the effect of correlated noises on SR for stationary analog inputs.

Although spike trains with small values of $M=1-3$ have been examined in Sec. III, we can make an analysis of spikes with larger $M$ or bursts, by using our method. In order to demonstrate its feasibility, we have made simulations for transient spikes with larger $M$. The upper curve of Fig. 16(a) expresses input $W_{\mathrm{i}}(t)$ with a $M=7$ spike train whose firing times are $t_{\mathrm{i} m}=100,115,130,145,160,180$, and $200 \mathrm{~ms}$, and ISIs are $T_{\mathrm{i}}=15$ and $20 \mathrm{~ms}$. Firings of 100 neurons in an ensemble is depicted by raster in Fig. 16(b), where the parameters of $g_{s}=0.06, D=1.0$ and $N=100$ are adopted. The lower curve in Fig. 16(a) shows its output $W_{\mathrm{o}}(t)$ averaged over the ensemble. We apply the WT to $W_{\mathrm{o}}(t)$ to get its WT coefficients and its WT decomposition, the latter being shown in Fig. 16(b). The $j=1$ and $j=2$ components are dominant. After the denoising, we get $\gamma=0.523$ and $S N R=18.6 \mathrm{~dB}$, which are comparable to those for $D=1.0$ with $M=1-3$ shown in Figs. 15(a) and 15(b). In our denosiging method given by Eqs.(39) and (45), we extract noises outside the $b$ region relevant to a cluster of spikes, but do not take account of noises between pulses. When a number of pulses $M$ and/or the ISI $T_{\mathrm{i}}$ become larger, a contribution from noises between pulses become considerable, and it is necessary to modify the denoising method such as to extract noises between pulses, for example, as given by

$$
\begin{aligned}
c_{j k}^{d n} & =c_{j k}, \quad \text { for } j \geq j_{c} \text { or } k_{\mathrm{L} m} \leq k \leq k_{\mathrm{U} m} \quad(m=1-M) \\
& =0 . \quad \text { otherwise }
\end{aligned}
$$

In Eq. (50) $k_{L m}$ and $k_{U m}$ are $m$ - and $j$-dependent lower and upper limits given by

$$
k_{\mathrm{Lm}}=2^{-j}\left(t_{\mathrm{om}} / T_{\mathrm{b}}-\delta b\right), \quad k_{\mathrm{Um}}=2^{-j}\left(t_{\mathrm{o} m} / T_{\mathrm{b}}+\delta b\right),
$$

where $t_{\mathrm{o} m}$ is the $m$-th firing time for the noise-free and supra-threshold input and $\delta b$ the margin of $b$.

An ensemble of neurons adopted in our model can be regarded as the front layer (referred to as the layer I here) of a synfire chain [77]: output spikes of the layer I are feed-forwarded to neurons on the next layer (referred to as the layer II) and spikes propagate through the synfire chain. The postsynaptic current of a neuron $\ell$ on the layer II is given by 


$$
I_{\ell}^{\mathrm{ps}}=(1 / N) \sum_{i=1}^{N} \sum_{m} w_{\ell i}\left(V_{a}-V_{s}\right) \alpha\left(t-\tau_{\ell i}-t_{\mathrm{oim}}\right)+I_{\ell}^{\mathrm{n}},
$$

where $w_{\ell i}$ and $\tau_{\ell i}$ are the synaptic coupling and delay time, respectively, for spikes to propagate from neuron $i$ on the layer I to neuron $\ell$ on layer II, $t_{\text {oim }}$ the $m$-th firing time of neuron $i$ on the layer I, and $I_{\ell}^{\mathrm{n}}$ noises. Transmission of spikes through the synfire chain depends on the couplings (excitatory or inhibitory) $w_{\ell i}$, the delay time $\tau_{\ell i}$ and noises $I_{\ell}^{\mathrm{n}}$. There are some discussions on the stability of spike transmission in a synfire chain. Quite recently Diesmann [78] has shown by simulations using IF models that the spike propagation with a precise timing is possible in fairly large-scale synfire chains with moderate noises.

By augmenting our neuron model including the coupling term given by Eq.(52) and tentatively assuming $w_{\ell i}=w=1.5$ and $I_{\ell}^{\mathrm{n}}=0$, we have calculated the transmission fidelity by applying our WT analysis to output signals on the layer II as well as those on the layer I. Calculated cross-correlation and SNR are shown in Fig. 17(a) and 17(b), respectively. In Fig. 17(a), $\gamma_{L}(\mathrm{~L}=\mathrm{I}, \mathrm{II})$ denotes the cross-correlation between the input and output signals on the layer $L$. Similarly, $S N R_{L}$ in Fig. 17(b) expresses the SNR of output signal on the layer $L$. Note that $\gamma_{I}$ and $S N R_{I}$ are nothing but $\gamma$ and SNR having been shown in Figs. 6(a) and 6(b). We note that the transmission fidelity on the layer II is better that that of the layer I because $\gamma_{I}<\gamma_{I I}$ and $S N R_{I}<S N R_{I I}$ at almost $D$ values. We have chosen $w=1.5$ such that neurons on the layer II can fire when more than $6 \%$ of neurons on the layer I fire. When we adopt smaller (larger) value of $w$, both $\gamma_{I I}$ and $S N R_{I I}$ abruptly increase at larger (smaller) $D$ value. However, the general behavior of the $D$ dependence of $\gamma_{I I}$ and $S N R_{I I}$ is not changed. This improvement of the transmission fidelity in the layer II than in the front layer I is expected to be partly due to the threshold-crossing behavior of $\mathrm{HH}$ neurons. It would be interesting to investigate the transmission of signals in a synfire chain by including SR of its front layer.

Our paper entirely relies on numerical simulations. We are currently trying to work on theoretical description of the result reported in this paper. Conventional approaches having been employed for a study of SR such as the rate-equation and linear-response theories [1]- [2], do not work on our case. Mato [26] adopted Gammaitoni's approach [79] for an analysis of his SR result with the continuous spike-train signals. It seems, however, not to be transposed directly to our case of transient spike-train signals even if our HH model is replaced by simpler IF model or threshold-crossing model. Its analytical study is left as our future problem.

To summarize, the response of an ensemble of neurons to transient spike trains has been discussed with the use of the WT. Although the transmission of sub-threshold transient inputs is not reliable in a single neuron, it is much improved in a large-scale ensemble of neurons with a weak noise (SR effect). It is also shown that this noise does not significantly degrade the transmission fidelity for supra-threshold inputs in the large-scale ensemble. A population of neurons plays an essential role in SR for transient inputs.

\section{ACKNOWLEDGEMENTS}

This work is partly supported by a Grant-in-Aid for Scientific Research from the Japanese Ministry of Education, Culture, Sports, Science and Technology. 


\section{REFERENCES}

[1] L. Gammaitoni, P. Hännggi, P. Jung, and F. Marchesoni, Rev. Mod. Phys. 70, 223 (1998).

[2] V. S. Anishchenko, A. B. Neiman, F. Moss and L. Schimansky-Geier, Soviet Phys.Uspekhi 42, 7 (1999).

[3] A. R. Bulsara, T. C. Elston, C. R. Doering, and K. Lindenberg, Phys. Rev. E 53, 3958 (1996).

[4] H. E. Plesser and S. Tanaka, Phys. Lett. A 225, 228 (1994).

[5] T. Shimokawa, K. Pakdaman, and S. Sato, Phys. Rev. E 59, 3427 (1999).

[6] A. Longtin, J. Stat. Phys. 70, 309 (1993).

[7] K. Wiesenfeld, D. Pierson, E. Pantazelou, C. Dames, and F. Moss, Phys. Rev. Lett. 72, 2125 (1994).

[8] A. Longtin and D. R. Chialvo, Phys. Rev. Lett. 81, 4012 (1994).

[9] S. Lee and S. Kim, Phys. Rev. E 60, 826 (1999).

[10] T. Shimokawa, A. Rogel, K. Pakdaman, and S. Sato, Phys. Rev. E 59, 3461 (1999).

[11] B. Lindner and L. Schimansky-Geier, Phys. Rev. Lett. 86, 2934 (2001).

[12] J. J. Collins, C. C. Chow and T. T. Imhoff, Nature 376, 236 (1995).

[13] T. Kanamaru, T. Horita, and Y. Okabe, Phys. Rev. E 64, 31908 (2000).

[14] N. G. Stocks, and R. Mannella, Phys. Rev. E 64, 30902 (2001).

[15] X. Pei, L. Wilkens, and F. Moss, Phys. Rev. Lett. 77, 4679 (1996).

[16] S. Tanabe, S. Sato, and K. Pakdaman, Phys. Rev. E 60, 7235 (1999).

[17] Y. Wang, D. T. W. Chik, and Z. D. Wang, Phys. Rev. E 61, 740 (2000).

[18] F. Liu, B. Hu, and W. Wang, Phys. Rev. E 63, 31907 (2000).

[19] J. K. Douglass, L. Wilkens, E. Pantazelou, and F. Moss, Nature 365, 337 (1993).

[20] X. Pei, L. A. Wilkens, and F. Moss, J. Neurophysiol. 76, 3002 (1996).

[21] J. E. Levins and J. P. Miller, Nature 380, 165 (1996).

[22] B. J. Gluckman, J. I. Netoff, E. J. Neel, W. L. Ditto, M. L. Spano, and S. J. Shiff, Phys. Rev. Lett. 77, 4098 (1996).

[23] D. Nozaki, D. J. Mar, P. Grigg, and J. J. Collins, Phys. Rev. Lett. 82, 2402 (1999).

[24] F. Chapeau-Blondeau, X. Godivier, and N. Chambet, Phys. Rev. E 53, 1273 (1996).

[25] G. Deco and B. Schürmann, Physica D 117, 276 (1998).

[26] G. Mato, Phys. Rev. E 58, 876 (1998); ibid. 59, 3339 (1999).

[27] J. J. Collins, C. C. Chow and T. T. Imhoff, Phys. Rev. E 52, R3321 (1995); ibid. 54, 5575 (1996).

[28] R. Fakir, Phys. Rev. E 57, 6996(1998); ibid. 58, 5175 (1998).

[29] R. D. Traub, J. G. R. Jefferys and M. A. Whittington: Fast Oscillations in Cortical Circuits (MIT press, Cambridge, 1999).

[30] W. E. Sullivan and M. Konishiki: J. Nerosci. 393, 268 (1998).

[31] G. Rose and W. Heilingenberg: Nature 318, 178 (1985).

[32] For reviews on the WT and its application see; N. M. Astaf'eva: Physics-Usp. 39, 1085 (1996); I. M. Dremin, O. V. Ivanov, and V. A. Nechitailo: hep-ph/0101182.

[33] V. J. Samar, A. Bopardikar, R. Rao and K. Swartz: Brain and Language 66, 7 (1999).

[34] S. Blanco, C. E. D’Attellis, S. I. Isaacson, O. A. Rosso, and R. O. Sirne: Phys. Rev. E 54, 6661 (1996). 
[35] S. Blanco, A. Figliola, R. Q. Quiroga, O. A. Rosso, and E. Serrano: Phys. Rev. E 57, 932 (1998).

[36] J. Ratz, L. Dickerson, and B. Turetsky: Brain and Language 66, 61 (1999).

[37] L. J. Trejo, and M. J. Shensa: Brain and Language 66, 89 (1999).

[38] T. Demiralp, A. Ademoglu, M. Schürmann, C. Başar-Eroglu, and E. Başar: Brain and Language 66, 108 (1999).

[39] T. Demiralp, J. Yordanova, V. Kolev, A. Ademoglu, M. Devrin, and V. J. Samar: Brain and Language 66, 29 (1999).

[40] O. A. Rosso, S. Blanco, J. Yordanova, V. Kolev, A. Figliola, M. Schürmann, E. Başar: J. Neurosci. Methods 105, 65 (2001).

[41] E. Hulata, R. Segev, Y. Shapir, M. Benveniste, and E.Ben-Jacob: Phys. Rev. Lett. 85, $4637(2000)$.

[42] J. C. Letelier and P. P. Weber: J. Neurosci. Methods 101, 93 (2000).

[43] G. Zouridakis, and D. C. Tam: Comput. Biol. Methods 27, 9 (1997).

[44] D. Stratimirović, S. Milosević, S. Blesić and M.Ljubiavljević: Physica A 291, 13 (2001).

[45] H. Hasegawa, E-print: cond-mat/0109444.

[46] A. Destexhe, Z. F. Mainen, and T. J. Sejnowski, in The Handbook of Brain Theory and Neural Networks', ed. M. A. Arbib. MIT press, Cambridgr 1998, p956

[47] R. G. Smith, in The Handbook of Brain Theory and Neural Networks', ed. M. A. Arbib. MIT press, Cambridgr 1998, p816

[48] M. N. Shadlen and W. T. Newsome, Curr. Opin.Nurobiol. 4, 569 (1994).

[49] H. Hasegawa, (unpublished).

[50] A. L. Hodgkin and A. F. Huxley, J. Physiol. 117, 500 (1952).

[51] E. A. Bartnik, K. J. Blinowska, and P. J. Durks, Biol. Cybem. 67, 175 (1992).

[52] O. Bertrand, J. Bohorquez, and J. Pemier, IEEE Trans, Biomed, Eng. 41, 77 (1994).

[53] D. L. Donoho, I. M. Johnstone, and B. W. Silverman, IEEE Trans, Inform. Theory 41, 613 (1995).

[54] R. Q. Quiroga, Physica D 145, 278 (2000).

[55] N. G. Stocks, Phys. Rev. Lett. 84, 2310 (2000); Phys. Rev. E 63, 41114 (2001).

[56] H. Hasegawa, Phys. Rev. E 61, 718 (2000); ibid. 62, 1456 [E]; Bull. Tokyo Gakugei Univ. Ser. 4, 53, 31 (2001).

[57] Z. F. Mainen, and T. J. Sejnowsky, Reliability of spike timing in neocortical neirons Science 268, 1503 (1995).

[58] F. Rieke, D. Warland, R. Steveninck and W. Bialek: Exploring the Neural Code (MIT press, Cambridge, 1996).

[59] R. C. deCharms: Proc. Natl. Acad. Sci USA 95, 15166 (1998).

[60] J. J. Eggermont: Neurosci. Biobehav. Rev, 22, 355 (1998).

[61] W. M. Ursey and R. C. Reid: Annu. Rev. Physiol. 61, 435 (1999).

[62] R. C. deCharms and A. Zador: Ann. Rev. Neurosci. 23, 613 (2000).

[63] A. Pouget, P. Dayan and R. Zemel: Nature Neurosci. 1, 125 (2000).

[64] A. D. Andrian, J. Physiol. (London) 61, 49 (1926).

[65] C. E. Carr, W. Heiligenberg and G. J. Rose, J. Neurosci. 6, 107 (1986).

[66] R. Eckhorn, R. Bauer, W. Jordan, M. Brosch, W. Kruse, M. Munk, and H. J. Reitboeck, Biol. Cybern. 60 121, (1988)

[67] C. M. Gray and W. Singer, Proc. Natl. Acad. Sci. (USA) 86, 1698 (1989). 
[68] E. T. Rolls and M. J. Tovee, Proc. Roy. Soc. B 257 9, (1994)

[69] S. Thorpe, D. Fize and C. Marlot, Nature (London) 381, 520 (1996).

[70] J J Hopfield: Nature 376, 33 (1995).

[71] D. Horn and S. Levanda: Neural Comput. 10, 1705 (1998).

[72] R. van Rullen and S. J. Thorpe: Neural Comput. 13, 1255 (2001).

[73] C. M. Gray and W. Singer: Proc. Natl. Acad. Sci. USA 86, 1698 (1989).

[74] R. C. deCharmes and M. M. Merzenich: Nature 381, 610 (1996).

[75] R. D. Traub, M. A. Whittington, J. G. R. Jefferys: Neural Comput. 9, 1251 (1997).

[76] N. Hatsopoulas, C. L. Ojakangas, L. Paninski and J. P. Donohue: Proc. Natl. Acad. Sci. USA 95, 15706 (1998).

[77] M. Abeles, H. Bergman, E. Margalit, and E. Vaadia, J. Neurophys. 70, 1629 (1993).

[78] M. Diesmann, M. Gewaltig, and A. Aertsen, Nature 402, 529 (1999).

[79] L. Gammaitoni, Phys. Rev. E 52, 4691 (1995). 


\section{FIGURES}

FIG. 1. The time dependence of (a) the $M=1$ input pulse $\left(U_{\mathrm{i}}\right),(\mathrm{b})$ the postsynaptic current $\left(I_{1}=I_{1}^{\mathrm{ps}}+I_{1}^{\mathrm{n}}\right)$, (c) the membrane potential $\left(V_{1}\right)$, and $(\mathrm{d})$ input $\left(W_{\mathrm{i}}\right)$ and $(\mathrm{e})$ output signals $\left(W_{\mathrm{o}}\right)$ within the time bin of $T_{\mathrm{b}}=5 \mathrm{~ms}$ for $D=0.5, g_{s}=0.06$ and $N=500$.

FIG. 2. Rasters showing firings in ensemble neurons for (a) $D=0.5$ and (b) $D=1.0$ with $M=1, g_{s}=0.06$ and $N=500$.

FIG. 3. (a) The input signal $W_{\mathrm{i}}(t)$ (uppermost frame) for $M=1$, and its WT decomposition: $f=\sum_{j=1}^{5} f_{j}$. (b) Its WT expansion coefficients $c_{j k}$. Curves of $f_{j}$ for $j \geq 2$ and of $f$ in (a), and those of $c_{j k}$ for $j \geq 2$ in (b) are successively shifted upward by 1.0. Upper horizontal scale expresses $b T_{\mathrm{b}}$ in units of ms.

FIG. 4. (a) The output signal $W_{\mathrm{o}}(t)$ (uppermost frame) for $D=0.5$ and $M=1$, and its WT decomposition: $f=\sum_{j=1}^{5} f_{j}$. (b) Its WT expansion coefficients $c_{j k}$. Solid and dashed curves denote the original and denoising results, respectively. Same as in Fig. 3.

FIG. 5. (a) The output signal $W_{\mathrm{o}}(t)$ (uppermost frame) for $D=1.0$ and $M=1$, and its WT decomposition: $f=\sum_{j=1}^{5} f_{j}$. (b) Its WT expansion coefficients $c_{j k}$. Solid and dashed curves denote the original and denoising results, respectively. Same as in Fig. 3.

FIG. 6. The $D$ dependence of $\gamma$ (circles), $\Gamma_{\text {oo }}$ (triangles) and $\Gamma$ (squares) for $M=1, g_{s}=0.06$ and $N=500$.

FIG. 7. The $\delta b$ dependence of SNR of output signals for (a) $D=0.5$ and (b) $D=1.0$ with $j_{c}=2$ (circles), 3 (triangles) and 4 (squares) for $M=1, g_{s}=0.06$ and $N=500$, the denoising condition being given by Eqs.(39) and (45).

FIG. 8. (a) The cross-correlation $\gamma$ and (b) SNR as a function of $D$ for $M=1$ and $g_{s}=0.06$ with $N=1,10,100$ and 500. Results for $N=1(N=10)$ are averaged values of 100 (10) trials and their RMS are shown by error bars. Results of (a) and (b) for $N=10,100$ and 500 are successively shifted upward by 1.0 and 20, respectively.

FIG. 9. (a) The cross-correlation $\gamma$ and (b) SNR of 100 trials for single $(N=1)$ neurons with $g_{s}=0.06$ and $M=1$.

FIG. 10. The $g_{s}$ dependence of the cross-correlation $\gamma$ and SNR for $N=500$ and $M=1$, Dot, dashed and solid curves denoting the results of $D=0.0,0.5$ and 1.0, respectively. The arrow expresses the threshold $g_{s}$ value. 
FIG. 11. The $g_{s}$ dependence of the cross-correlation $\gamma$ and SNR for $N=1$ and $M=1$ : Dot, dashed and solid curves denote the results of $D=0.0,0.5$ and 1.0, respectively, and error bars express RMS values for 100 trials.

FIG. 12. Raster showing firings in ensemble neurons for (a) $M=2$ and (b) $M=3$ with $D=1.0, g_{s}=0.06$ and $N=500$.

FIG. 13. (a) The output signal $W_{\mathrm{o}}(t)$ (uppermost frame) for $D=1.0$ and $M=2$, and its WT decomposition: $f=\sum_{j=1}^{5} f_{j}$. (b) Its WT expansion coefficients $c_{j k}$. Solid and dashed curves denote the original and denoising results, respectively. Same as in Fig. 3.

FIG. 14. (a) The output signal $W_{\mathrm{o}}(t)$ (uppermost frame) for $D=1.0$ and $M=3$, and its WT decomposition: $f=\sum_{j=1}^{5} f_{j}$. (b) Its WT expansion coefficients $c_{j k}$. Solid and dashed curves denote the original and denoising results, respectively. Same as in Fig. 3.

FIG. 15. (a) The cross-correlation $\gamma$ and (b) SNR as a function of $D$ for $M=1$ (circles), $M=2$ (squares) and $M=3$ (triangles).

FIG. 16. (a) The input spike train $W_{\mathrm{i}}(t)$ (upper curve) consisting of $M=10$ pulses, and its output signal $W_{\mathrm{o}}(t)$ (lower curve) for $g_{s}=0.06, D=1.0$ and $N=100$. (b) Raster showing firings of 100 neurons. (c) The WT decomposition of $W_{\mathrm{o}}(t)$, results for $j=2,3,4$ and 5 being successively shifted upward by 0.2 .

FIG. 17. (a) The $D$ dependence of the cross-correlation $\gamma_{I}$ (filled circles) and $\gamma_{I I}$ (open circles), and (b) that of $S N R_{I}$ (filled circles) and $S N R_{I I}$ (open circles) for $M=1$; note that $S N R_{I I}=\infty$ for $0.3<D<0.6$ (see text). 
This figure "fig1-5.gif" is available in "gif" format from: http://arxiv.org/ps/cond-mat/0111546v1 
This figure "fig6-9.gif" is available in "gif" format from: http://arxiv.org/ps/cond-mat/0111546v1 
This figure "fig10-12.gif" is available in "gif" format from: http://arxiv.org/ps/cond-mat/0111546v1 
This figure "fig13-17.gif" is available in "gif" format from: http://arxiv.org/ps/cond-mat/0111546v1 